\title{
Modeling fibrosis using fibroblasts isolated from scarred rat vocal folds
}

\author{
Yo Kishimoto ${ }^{1,3}$, Ayami Ohno Kishimoto ${ }^{1,4}$, Shuyun Ye $^{2}$, Christina Kendziorski ${ }^{2}$ and Nathan V Welham ${ }^{1}$
}

Following injury, pathologically activated vocal fold fibroblasts (VFFs) can engage in disordered extracellular matrix (ECM) remodeling, leading to VF fibrosis and impaired voice function. Given the importance of scar VFFs to phenotypically appropriate in vitro modeling of VF fibrosis, we pursued detailed characterization of scar VFFs obtained from surgically injured rat VF mucosae, compared with those obtained from experimentally naïve, age-matched tissue. Scar VFFs initially exhibited a myofibroblast phenotype characterized by increased proliferation, increased Col1a1 transcription and collagen, type I synthesis, increased Acta2 transcription and $a$-smooth muscle actin synthesis, and enhanced contractile function. These features were most distinct at passage 1 (P1); we observed a coalescence of the scar and naïve VFF phenotypes at later passages. An empirical Bayes statistical analysis of the P1 cell transcriptome identified 421 genes that were differentially expressed by scar, compared with naïve, VFFs. These genes were primarily associated with the wound response, ECM regulation, and cell proliferation. Follow-up comparison of P1 scar VFFs and their in vivo tissue source showed substantial transcriptomic differences. Finally, P1 scar VFFs responded to treatment with hepatocyte growth factor and transforming growth factor- $\beta 3$, two biologics with reported therapeutic value. Despite the practical limitations inherent to working with early passage cells, this experimental model is easily implemented in any suitably equipped laboratory and has the potential to improve the applicability of preclinical VF fibrosis research.

Laboratory Investigation (2016) 96, 807-816; doi:10.1038/labinvest.2016.43; published online 25 April 2016

Fibroblasts, the most abundant cells of many connective tissues, regulate tissue homeostasis under quiescent conditions and drive regeneration and structural reorganization during wound healing. ${ }^{1}$ Research conducted over the past few decades has highlighted the role of resident and migratory fibroblasts in initiating and sustaining chronic fibrosis in key organ systems, such as the kidney, liver, lung, and skin. ${ }^{2-7}$ This body of work has shown that fibroblast phenotype varies as a function of both host organ ${ }^{8}$ and the physiologic (or pathophysiologic) state of the organ. ${ }^{4}$ Consequently, although all fibroblasts share certain defining features, the phenotype of one fibroblast subtype is not necessarily predictive of another.

Vocal fold fibroblasts (VFFs) populate the lamina propria of the VF mucosa and are responsible for synthesizing its extracellular matrix (ECM). ${ }^{9}$ This ECM has exquisite viscoelasticity that supports high-frequency, self-sustained tissue oscillation for voice production. ${ }^{10,11}$ In response to sustained inflammatory and profibrotic stimuli, however, activated VFFs can pathologically remodel the ECM, resulting in dense and disorganized collagen and fibronectin, along with reduced elastin, decorin, and hyaluronic acid. ${ }^{12-14}$ Such ECM protein and glycan alterations are the hallmarks of chronic VF scar and typically result in viscoelastic deterioration and intractable voice impairment. ${ }^{15}$

One approach to treating chronic VF scar is to directly manipulate VFF behavior using an antifibrotic therapy. Several preclinical in vitro studies have pursued such an approach using biologics, ${ }^{16,17}$ biomaterials, ${ }^{18}$ and cell-based interventions; ${ }^{19}$ however, this work has typically been conducted using naïve VFFs under the assumption that the treatment-response phenotype of these cells is generalizable to that of scar VFFs. This assumption may be incorrect: recent data generated using scar VFFs isolated from two ferrets ${ }^{20,21}$ and from a single human patient ${ }^{22-24}$ suggest that, compared with naïve cells, scar VFFs exhibit different growth kinetics, cytokine profiles and ECM-synthesis rates, as well as

\footnotetext{
${ }^{1}$ Department of Surgery, Division of Otolaryngology, University of Wisconsin School of Medicine and Public Health, Madison, WI, USA and ${ }^{2}$ Department of Biostatistics and Medical Informatics, University of Wisconsin School of Medicine and Public Health, Madison, WI, USA

Correspondence: Dr N Welham, PhD, Department of Surgery, Division of Otolaryngology, University of Wisconsin School of Medicine and Public Health, K4/723 CSC, 600 Highland Avenue, Madison 53792, WI, USA.

E-mail: welham@surgery.wisc.edu

${ }^{3}$ Present address: Department of Otolaryngology, Kyoto University School of Medicine, Kyoto 606-8507, Japan.

${ }^{4}$ Present address: Department of Radiology, Kyoto City Hospital, Kyoto 604-8845, Japan.

Received 1 August 2015; revised 2 February 2016; accepted 19 February 2016
} 
enhanced lipopolysaccharide responsiveness and contractile myofibroblastic features. These observations, which are consistent with findings in the wider fibroblast literature, ${ }^{4}$ highlight the value of conducting fibrosis-related in vitro work with cells obtained from scarred tissue.

Given the apparent importance of scar VFFs to phenotypically appropriate in vitro modeling of VF fibrosis, and the paucity of current data in this area, we pursued detailed characterization of scar VFFs obtained from surgically injured rat VF mucosae, compared with VFFs from experimentally naïve, age-matched tissue. The rat is a well-validated model in VF biology and its in vivo scar phenotype has been extensively described. ${ }^{13,25-27}$ We used a larger set of independent biological replicates than has been previously reported and examined cell phenotypes across serial-culture passages beginning at first passage. We first evaluated overall cell proliferation and apoptosis, collagen production, evidence of myofibroblast differentiation, and contractile function. We then measured transcriptomic differences between scar and naïve VFF, as well as between early passage scar VFFs and their in vivo tissue source. ${ }^{28}$ Finally, we examined scar VFF responsiveness to hepatocyte growth factor (HGF) and transforming growth factor- $\beta 3$ (TGF- $\beta 3$ ), two biologics with reported therapeutic value. ${ }^{17,29,30}$

\section{MATERIALS AND METHODS Animals}

Fischer 344 male rats (Charles River, Wilmington, MA, USA) were used for all experiments. Bilateral VF injuries were created in 120-day-old rats using a surgical stripping procedure and tissues were harvested following a 60-day scar maturation period. ${ }^{13}$ Experimentally naïve control tissues were harvested from age-matched (180-day-old) rats. All animal experiments were conducted in accordance with the Public Health Service Policy on Humane Care and Use of Laboratory Animals, and the Animal Welfare Act (7 U.S.C. et seq.); the Institutional Animal Care and Use Committee of the University of Wisconsin-Madison approved all protocols.

\section{Cell Isolation and Culture}

Explant cell culture was conducted using previously reported techniques for primary VFF. ${ }^{31,32}$ Scarred and naïve rat VF mucosae were obtained from freshly harvested rat larynges using a dissection microscope. Each pair of bilateral mucosae was minced in a $10 \mathrm{~cm}$ culture dish and immersed in DMEM supplemented with 10\% FBS, antibiotics and antimycotics (all culture reagents from Sigma-Aldrich, St Louis, MO, USA). Cells were cultured at $37^{\circ} \mathrm{C}$ in $5 \% \mathrm{CO}_{2}$ and medium was changed twice weekly. Outgrown primary cells were trypsinized and passaged into fresh $10 \mathrm{~cm}$ culture dishes 14-21 days after initial explant plating (mean yield, $12 \times 10^{4}$ VFF; range, $\left.8 \sim 14.5 \times 10^{4} \mathrm{VFF}\right)$. Subsequent passaging was performed when cells were $80 \%$ confluent: VFFs were trypsinized, counted, and plated in $10 \mathrm{~cm}$ culture dishes at a density of $2-3 \times 10^{5}$ cells/dish (split ratio, 1:3-1:5). VFF cultures were maintained through $\mathrm{P} 4$.

\section{Cell Proliferation Analysis}

VFFs were plated in 24-well plates at a density of $10^{4}$ cells/well and cultured. After 6 days, cells were trypsinized and counted using a hematocytometer. All counts were performed in technical duplicate. Population doubling time was calculated as $2^{N}=C_{f} / C_{i}$, where $N$ denotes doubling time, $C_{f}$ denotes the final cell count at time of harvest, and $C_{i}$ denotes the initial cell count at time of seeding. ${ }^{33}$

\section{Gel Contraction Assay}

The gel contraction assay was performed as previously reported. ${ }^{34}$ In brief, 24 -well plates were pre-incubated with PBS containing 1\% BSA (Sigma-Aldrich) for at least $1 \mathrm{~h}$ at $37^{\circ} \mathrm{C}$, washed twice with PBS, and air dried. Rat tail collagen, type I (BD Biosciences, Franklin Lakes, NJ, USA) was prepared to a final concentration of $2.4 \mathrm{mg} / \mathrm{ml}$ in PBS and seeded with $12.5 \times 10^{4} \mathrm{P} 1 \mathrm{VFFs} / \mathrm{ml}$. Four hundred $\mu$ l of the collagen/cell mixture was added to each culture well and polymerized for $30 \mathrm{~min}$ at $37^{\circ} \mathrm{C}$. Culture medium was added to each well and the gels were detached from the plate surface with gentle shaking. VFF culture was performed for $72 \mathrm{~h}$ and gels were imaged at $0,24,48$, and $72 \mathrm{~h}$. Polymerized gels containing no cells were used as negative controls. Gel area was calculated as $A=d_{\max } d_{\min } \pi / 4$, where $A$ denotes area, $d_{\max }$ denotes maximum gel diameter, and $d_{\min }$ denotes minimum gel diameter.

\section{Growth Factor Treatment Experiments}

P1 scar VFFs were plated in six-well plates at a density of $2 \times 10^{4}$ cells/well and cultured until $80 \%$ confluent. Following $24 \mathrm{~h}$ of serum starvation, cells were treated with $0,1,10$, or $100 \mathrm{ng} / \mathrm{ml}$ HGF (PeproTech, Rocky Hill, NJ, USA); or with 0, 1,10 , or $100 \mathrm{ng} / \mathrm{ml}$ TGF- $\beta 3$ (R\&D Systems, Minneapolis, $\mathrm{MN}$, USA). Cells were harvested $24 \mathrm{~h}$ post-treatment and processed for qRT-PCR.

\section{Immunocytochemistry}

P1 VFFs were placed on chamber slides, fixed with $4 \%$ paraformaldehyde, and permeabilized with $0.5 \%$ Triton X-100 (Sigma-Aldrich). Image-iT FX signal enhancer (Invitrogen, Carlsbad, CA, USA) was applied for $30 \mathrm{~min}$ to reduce nonspecific fluorescent signals. Blocking was performed using Block Ace (AbD Serotec, Raleigh, NC, USA). Cells were sequentially incubated with a primary antibody at room temperature (RT) for $1 \mathrm{~h}$, a relevant secondary antibody at RT for $1 \mathrm{~h}$, and counterstained with DAPI nuclear dye (MP Biomedicals, Santa Ana, CA, USA).

The primary antibodies used were: goat anti-collagen, type I (1:20; 1310-01, Southern Biotechnology, Birmingham, $\mathrm{AL}$, USA); rabbit anti- $\alpha$-SMA (1:100; ab5694, Abcam, Cambridge, MA, USA), rabbit anti-Ki-67 (1:50; ab16667, Abcam); and rabbit anti-CC3 (1:50; \#9969, Cell Signaling Technology, 
Danvers, MA, USA). The secondary antibodies used were: Alexa Fluor 594 goat anti-rabbit or donkey anti-goat IgG (1:400, A-11012 or A-11058; Invitrogen). Negative control sections exposed to the secondary antibody in the absence of the primary antibody revealed no immunosignals.

\section{RNA Isolation and qRT-PCR}

Total RNA was isolated from cells using the RNeasy Mini kit (Qiagen, Valencia, CA, USA) according to the manufacturer's instructions. Samples were treated with DNase I to eliminate contamination by genomic DNA (Ambion, Austin, TX, USA). RNA yield and purity were evaluated by measuring concentration and $\mathrm{OD}_{260: 280}$ values with a NanoDrop ND-1000 spectrophotometer (NanoDrop, Wilmington, DE, USA) and by visualizing $18 \mathrm{~S}$ and $28 \mathrm{~S}$ rRNA bands on a $1 \%$ agarose gel. cDNA was generated by reverse transcription using TaqMan reagents (Applied Biosystems, Foster City, CA, USA) according to the manufacturer's instructions.

qRT-PCR amplification was performed using the following rat-specific commercial primers (QuantiTect; Qiagen): QT01615901 (Acta2); QT00370622 (Col1a1); QT01083537 (Col3a1); QT00179333 (Fn1); QT00195958 (Sdha). Reactions were performed on a 7500 Fast Real-Time PCR system (Applied Biosystems) using the QuantiTect SYBR Green PCR kit (Qiagen). Each $25 \mu \mathrm{l}$ total volume reaction contained $12.5 \mu \mathrm{l} 2 \times$ QuantiTect master mix, $2.5 \mu \mathrm{l} 10 \times$ QuantiTect primer assay, and $10 \mu \mathrm{l} \mathrm{cDNA}$ template diluted in nucleasefree $\mathrm{H}_{2} \mathrm{O}$. The cycling program was as follows: initial activation at $95^{\circ} \mathrm{C}$ for $15 \mathrm{~min}$, followed by 40 cycles of $94^{\circ}$ $\mathrm{C}$ for $15 \mathrm{~s}, 55^{\circ} \mathrm{C}$ for $30 \mathrm{~s}$, and $72{ }^{\circ} \mathrm{C}$ for $30 \mathrm{~s}$. All PCR reactions were performed in technical duplicate. Relative mRNA expression was calculated using the standard curve method; all values were normalized against reference gene $S d h a .{ }^{35}$

\section{Microarrays}

Biotinylated antisense cRNA was prepared by single round in vitro amplification of $0.9 \mu \mathrm{g}$ input RNA using the MessageAmp II-Biotin Enhanced aRNA kit (Ambion) according to the manufacturer's instructions (the in vitro transcription reaction was performed at $37^{\circ} \mathrm{C}$ for $14 \mathrm{~h}$ ). Poly-A RNA controls (Affymetrix, Santa Clara, CA, USA) were spiked into each reaction. Fragmented cRNA sample quality was confirmed using $2 \%$ agarose gel electrophoresis and Agilent 2100 Bioanalyzer analysis. Samples were hybridized to Affymetrix GeneChip Rat Genome 2302.0 arrays at $45^{\circ} \mathrm{C}$ for $16 \mathrm{~h}$. Post-processing was performing using the GeneChip Fluidics Station 450, arrays were scanned using the GC3000 G7 scanner, and fluorescent intensity data were background-corrected and extracted using Expression Console software (Affymetrix). All hybridization, post-processing and scanning procedures were performed according to Affymetrix protocols; all control parameters were within the manufacturer's guidelines. Microarray data have been deposited with the Gene Expression Omnibus (http://www.ncbi. nlm.nih.gov/geo/) under accession number GSE62204.

\section{Western Blotting}

VFFs were lysed in RIPA buffer (Thermo Fisher, Waltham, MA, USA) and total protein was extracted according to the manufacturer's instructions. Protein concentration was measured using the Pierce BCA assay kit (Thermo Fisher) according to the manufacturer's instructions. SDS-PAGE was performed using a $10 \%$ acrylamide gel (Bio-Rad, Hercules, CA, USA) with $30 \mu \mathrm{g}$ total protein load per lane. Following transfer, PVDF membranes were treated with 5\% nonfat dry milk at RT for $1 \mathrm{~h}$ to prevent nonspecific binding, and then incubated with the following primary antibodies at $4{ }^{\circ} \mathrm{C}$ overnight: rabbit anti- $\alpha$-SMA (1:500; ab5694, Abcam); goat anti-collagen, type I $(1: 400 ; 1310-01$, Southern Biotechnology); mouse anti-Sdha (1:3000; ab14715, Abcam). Blots were detected using relevant HRP-conjugated goat antimouse, goat-anti-rabbit and rabbit anti-goat IgG secondary antibodies (1:5000; 170-5047, 170-5046 and 172-1034, Bio-Rad) at RT for $1 \mathrm{~h}$ and the Immun-Star WesternC chemiluminesence kit (Bio-Rad). Images were captured using the ImageQuant LAS 4000 mini biomolecular imager (GE Healthcare, Piscataway, NJ, USA). Densitometric analysis was performed using Imagej; ${ }^{36}$ band densities were normalized against those of reference protein Sdha. Analysis of collagen, type I was performed using both bands (corresponding to the protein's $\alpha-1$ and $\alpha-2$ chains).

\section{Statistical Analyses}

All experiments were performed with $n=3-5$ independent biological replicates per condition; the sample size for each experiment is reported in the relevant figure legend. For each analysis, $n=2-5$ technical replicates were averaged and all statistical analyses were performed using biological replicates. Proliferation, qRT-PCR and western blot densitometric data were analyzed using two-way analysis of variance (ANOVA) with cell phenotype (scar $v s$ naïve) and culture passage as independent variables; qRT-PCR data from the growth factor treatment experiment were analyzed using one-way ANOVA; gel contraction data were analyzed using repeated-measures ANOVA. In all ANOVA models, if the omnibus F-test revealed a significant difference, planned pairwise comparisons were performed using Fisher's protected least significant difference method. Data were rank-transformed where needed to meet the equal variance assumptions of ANOVA.

Microarray data were analyzed within the $\mathrm{R}$ statistical computing environment. ${ }^{37}$ Affymetrix probe-level data were preprocessed using Robust Multi-Array Analysis (RMA), ${ }^{38}$ based on evidence of improved precision over default Affymetrix algorithms. ${ }^{39}$ Probes without a corresponding gene symbol were purged from all gene-level analyses. In cases where multiple probes corresponded to a single gene symbol, we calculated the average expression for each probe across arrays and selected the probe with the median average expression. In the case of an even number of matched probes, we selected the larger of the two median probe intensities. 
The resulting normalized data were clustered to check for consistency prior to formal analysis.

Expression analysis was performed using an empirical Bayes approach as implemented in the $\mathrm{R}$ package EBarrays. ${ }^{40}$ A lognormal-normal moderated variance (LNNMV) model was fit to the data; parameter estimates were obtained via 20 iterations of an expectation-maximization (EM) algorithm: in all cases, convergence was achieved after 10 iterations. Diagnostic testing of the LNN assumption in EBarrays was performed using quantile-quantile (QQ) plots of log intensity data $v s$ a standard normal distribution. We further used QQ plots to evaluate the assumption of a scaled inverse chi-square prior on the gene-specific variances used in the LNNMV model. The diagnostics showed no violations of model assumptions.

Using the output from EBarrays, we compared expression levels in the scar and naïve VFF conditions at $\mathrm{P} 1$, and further compared these in vitro data with a previously reported in vivo rat VF mucosal injury microarray data set. ${ }^{28}$ This pre-existing data set was generated using an identical rat strain and age at the time of injury, identical tissue harvest at 60 days postinjury, as well as identical RNA extraction, amplification, hybridization, and post-processing protocols. For all comparisons, thresholding was performed using $>0.95$ posterior probability of differential expression (DE), providing false discovery rate control at the $5 \%$ level.

Tests of enrichment via overrepresentation were conducted using the R package allez, ${ }^{41}$ the Gene Ontology (GO) data set, ${ }^{42}$ and genes identified as DE in the scar, compared with naïve, VFF condition at P1. Overrepresented GO terms required at least 25 distinct $\mathrm{DE}$ genes and a $Z$-score $>5$, and were further processed using the REViGO semantic similarity and term redundancy algorithm ${ }^{43}$ followed by Cytoscape 2.8.2. ${ }^{44}$ Heat maps summarizing expression data for DE gene lists of interest were generated using the Matrix2png utility. ${ }^{45}$

\section{RESULTS}

\section{Scar VFFs Exhibit Increased Proliferation at Early Passages}

We first compared the growth kinetics of primary VFFs isolated from naïve and scarred rat VF mucosae. Given the low density of VFFs in naïve rat VF mucosa $\mathrm{a}^{25}$ and a decision not to pool cells across biological replicates, we obtained insufficient cell yields at first passage to perform within-passage growth curve experiments. We therefore counted cells at the time of initial seeding and following 6 days of culture, and used these data to calculate population doubling times for VFFs at passage 1 (P1) through P4. Scar VFFs proliferated more rapidly (and exhibited correspondingly shorter doubling times) compared with naïve cells at P1-P3 $(P<0.05 ;$ Figure 1a). No significant differences were observed at $\mathrm{P} 4(P>0.05)$. These quantitative data were corroborated by immunocytochemistry (ICC) at P1 showing a greater number of scar VFF nuclei expressing

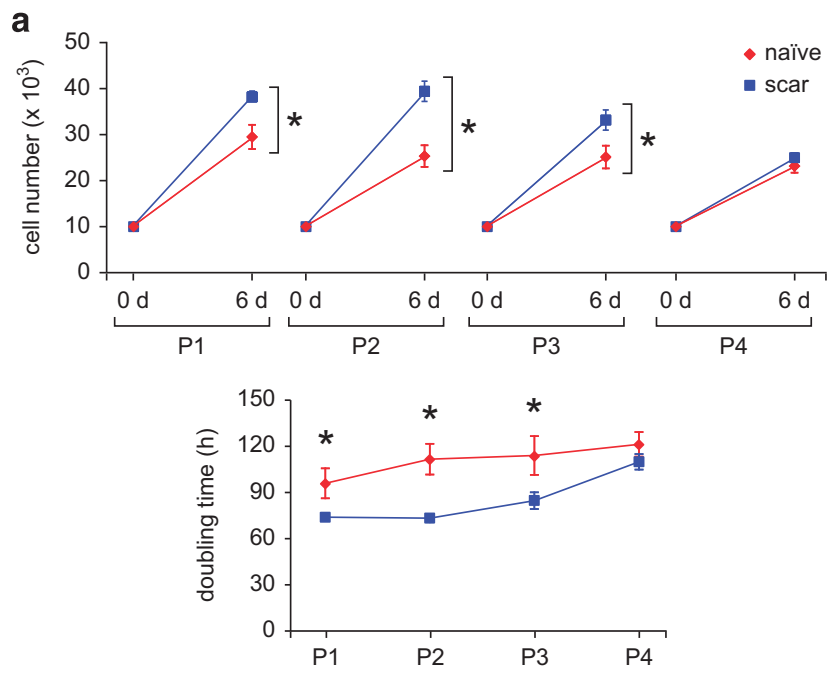

b
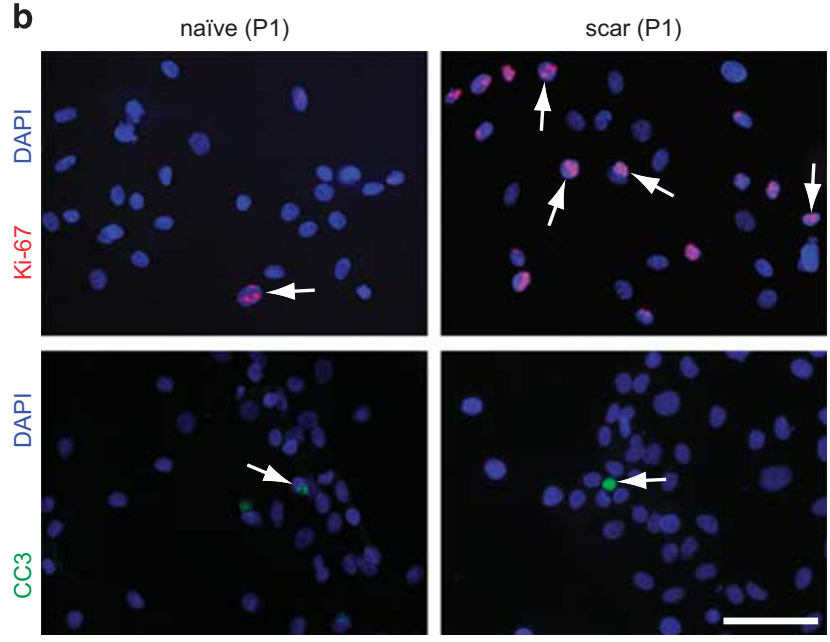

Figure 1 Scar VFFs exhibit increased proliferation at early passages. (a) Cell count data showing greater proliferation of scar VFFs compared with naïve cells after 6 days (d) incubation at P1-P3. Corresponding population doubling times (in hours (h)) are also shown. (b) Representative ICC data showing Ki-67 (red) and CC3 (green) expression in naïve and scar VFFs at P1. Nuclei are counterstained with DAPI (blue). Ki- $67^{+}$cells were more abundant in the scar, compared with naïve, condition; no difference was observed for CC3. White arrows indicate $\mathrm{Ki}^{-} 67^{+}$or $\mathrm{CC}^{+}$ fibroblast nuclei. All experiments were performed with $n=4$ biological replicates per condition. Data are presented as mean \pm s.e.m. ${ }^{*} P<0.05$ in a. Scale bar $=100 \mu \mathrm{m}$ in $\mathbf{b}$.

the proliferation marker Ki-67 (Figure 1b). The apoptosis marker cleaved caspase 3 (CC3) was rarely expressed by either VFF phenotype (Figure 1b).

\section{Scar VFFs Exhibit Increased Col1a1 Transcription and Collagen, Type I Synthesis at P1}

Disordered ECM is a defining feature of VF scar in vivo. ${ }^{12-14}$ We therefore examined the transcription of key ECM genes Colla1, Col3a1, and Fn1 in naïve and scar VFFs at P1-P4, using qRT-PCR. Collal transcription was upregulated in scar, compared with naïve, VFFs at P1 only $(P<0.05$; Figure $2 \mathrm{a})$; 

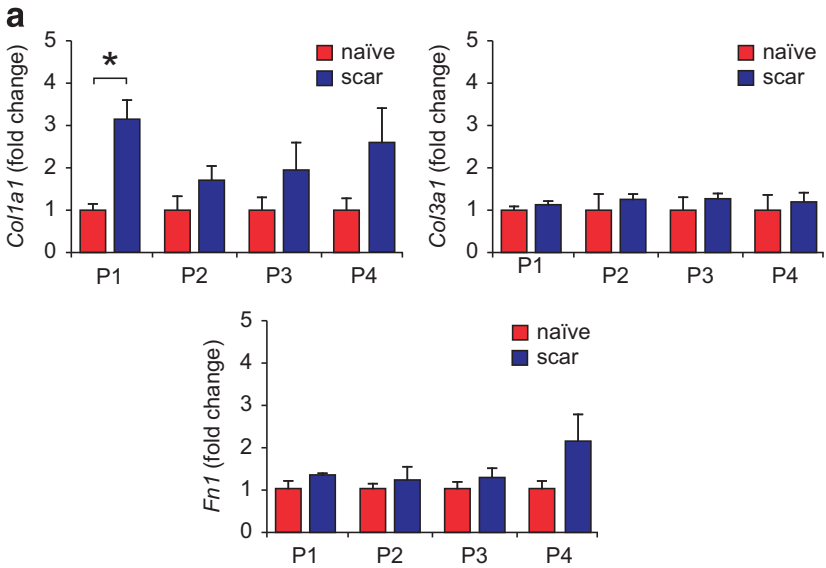

b
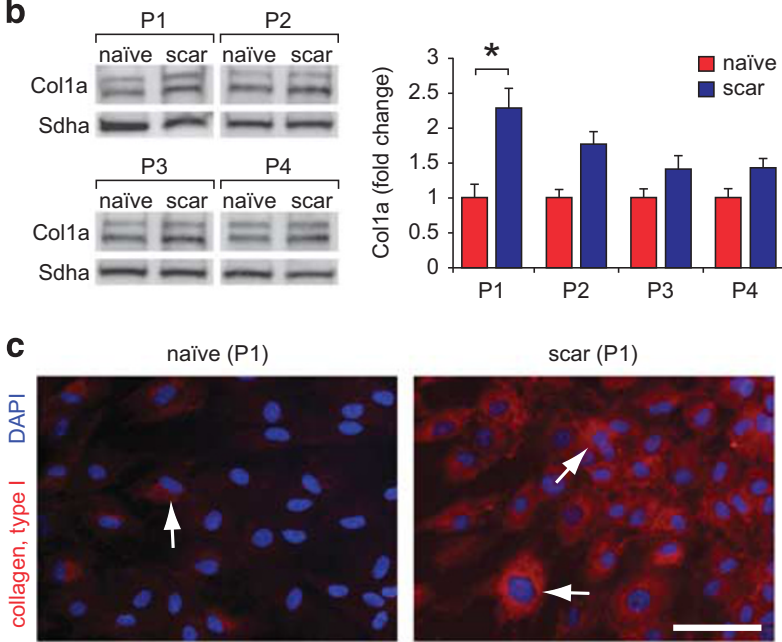

Figure 2 Scar VFFs exhibit increased Col1a1 transcription and collagen, type I synthesis at P1. (a) qRT-PCR-based analysis of Col1a1, Col3a1, and Fn1 transcription in naïve and scar VFFs at P1-P4. Col1a1 was significantly upregulated in scar VFFs at P1 only, whereas Col3a1 and Fn1 exhibited no significant differences at all passages. (b) Representative western blots showing Col1a abundance in naïve and scar VFFs (left panel; the double bands correspond to the protein's $a-1$ and $a-2$ chains). Sdha is shown as a loading control. The Sdha blots are identical to those shown in Figure $3 \mathrm{~b}$, as Col1a and $a$-SMA were probed on the same membranes. Corresponding densitometric analysis of Col1a abundance, normalized to Sdha (right panel). Col1a was upregulated in scar VFFs at P1 only. (c) Representative ICC data showing strong collagen, type I immunosignals (red) in scar, compared with naïve, VFFs at P1. Nuclei are counterstained with DAPI (blue). White arrows indicate collagen, type $\mathrm{I}^{+}$ fibroblasts. Quantitative data in $\mathbf{a}$ and $\mathbf{b}$ are presented as mean fold change \pm s.e.m. $v s$ the naïve VFF condition at the same passage. ${ }^{*} P<0.05$ in $\mathbf{a}$ and $\mathbf{b}$. All experiments were performed with $n=4$ biological replicates per condition. Scale bar $=100 \mu \mathrm{m}$ in c.

no significant differences were observed for Col3al and Fn1 at any passage $(P>0.05)$. We evaluated collagen, type I abundance at the protein level by western blotting for both $\alpha-1$ and $\alpha$-2 chains, confirming significant upregulation at P1 $(P<0.05$; Figure $2 \mathrm{~b})$ followed by tapering over subsequent passages. We further corroborated this finding using ICC performed on cells at P1 (Figure 2c).

\section{Scar VFFs Exhibit a Contractile Myofibroblast Phenotype at P1}

Fibroblasts are one of several cell populations that are capable of assuming a contractile myofibroblast phenotype during wound healing, ${ }^{5,46}$ including in the VF mucosa. ${ }^{26}$ Myofibroblasts are necessary for achieving wound closure and therefore have an important role in nonpathologic healing; however, unchecked myofibroblast activation can drive excess tissue contraction and ECM production, leading to chronic fibrosis and impaired function. ${ }^{4}$ To evaluate whether VFFs isolated from chronically scarred VF mucosae exhibit features of persistent myofibroblastic activation in vitro, we evaluated transcription of the myofibroblast marker Acta2 using qRT-PCR. Similar to our findings for the ECM gene Colla1, Acta2 transcription was upregulated at $\mathrm{P} 1$ only $(P<0.05$; Figure $3 \mathrm{a})$. We corroborated this finding at the protein level using western blotting and ICC and observed higher $\alpha$-smooth muscle actin ( $\alpha$-SMA) abundance in scar, compared with naïve, VFFs at $\mathrm{P} 1(P<0.05$; Figure $3 \mathrm{~b}$ and $\mathrm{c})$.

Based on these transcription and immunodetection data, we next evaluated the contractile ability of P1 VFFs when cultured in a collagen gel. Compared with naïve control, scar VFFs showed greater gel contraction capacity over $72 \mathrm{~h}$ $(P<0.05$; Figure $3 \mathrm{~d})$, consistent with having a functional myofibroblast phenotype at P1.

\section{Analysis of the Scar VFF Transcriptome}

Given our experimental data showing differences in the transcription of fibrosis-related genes by naïve and scar VFFs at $\mathrm{P} 1$, and the importance of comprehensive characterization of the scar VFF phenotype, we used expression microarrays to profile the scar VFF transcriptome. We prepared P1 naïve and scar VFFs for standard analysis using Affymetrix rat genome expression arrays, evaluated DE using an empirical Bayes approach, ${ }^{40}$ and conducted enrichment analysis using GO annotations. $^{42}$ A total of 598 probes, corresponding to 421 unique genes, were DE in the scar VFF condition compared with the naïve control condition. These 421 genes corresponded to enrichment of 73 GO terms (Supplementary Table S1): the majority (54) of enriched terms were associated with the biological process domain; fewer terms were associated with the cellular component (16) and molecular function (3) domains. Post-processing of these enrichment data using the REViGO semantic similarity and term redundancy algorithm ${ }^{43}$ highlighted biological process terms associated with cell division and proliferation, adhesion, and response to wounding; cellular component terms associated with the ECM and cell nucleus; and molecular function terms associated with the ECM and microtubule activity (Figure 4a).

We further examined the relative expression of DE genes associated with three representative GO terms of interest: response to wounding, ECM, and cell division (Figure 4b). Scar VFFs overexpressed a variety of transcripts across these functional categories, including products of the cytokine and chemokine genes Illa, Ccl11, and Cxcl5; the collagen family 


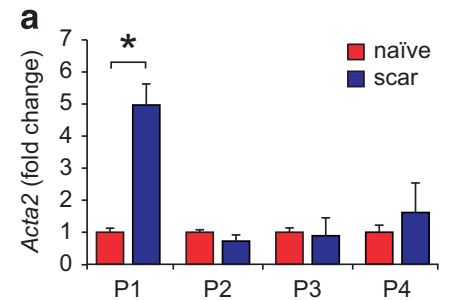

b
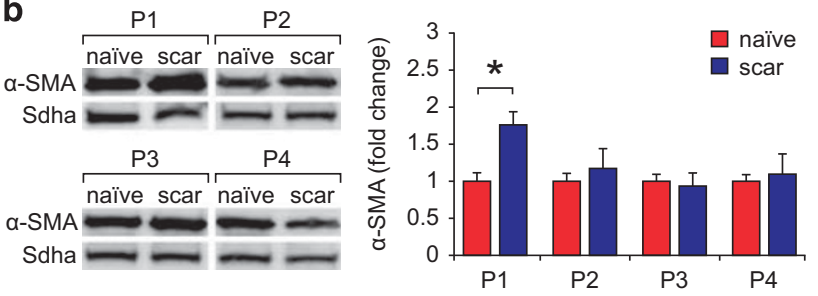

C
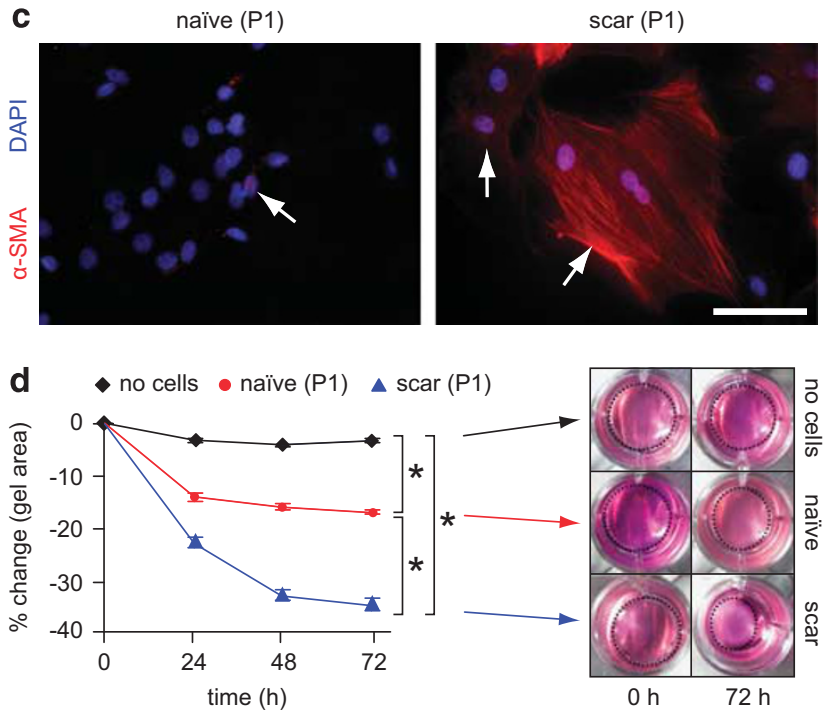

Figure 3 Scar VFFs exhibit a contractile myofibroblast phenotype at P1. (a) qRT-PCR data showing increased Acta2 transcription in scar VFFs compared with naïve cells at P1 only. (b) Representative western blots showing $a$-SMA abundance in naïve and scar VFFs (left panel). Sdha is shown as a loading control. The Sdha blots are identical to those shown in Figure $2 b$, as Col1a and $a$-SMA were probed on the same membranes. Corresponding densitometric analysis of $a$-SMA abundance, normalized to Sdha (right panel). $a$-SMA was upregulated in scar VFFs at P1 only.

(c) Representative ICC data showing strong $a$-SMA (red) immunosignals in scar, compared with naïve, VFFs at P1. Nuclei are counterstained with DAPI (blue). White arrows indicate $a-\mathrm{SMA}^{+}$(myo)fibroblasts. (d) Collagen gel contraction data showing greater area change in gels seeded with scar, compared with naïve, VFFs at P1. Quantitative data in $\mathbf{a}$ and $\mathbf{b}$ are presented as mean fold change \pm s.e.m. vs the naïve VFF condition at the same passage; gel contraction data in $\mathbf{d}$ are presented as percent change \pm s.e.m. vs the initial seeding $(0$ hour $(\mathrm{h}))$ condition. ${ }^{*} P<0.05$ in a, b, d. All experiments were performed with $n=4$ biological replicates per condition. Scale bar $=100 \mu \mathrm{m}$ in $\mathbf{c}$.

genes Col5a1, Col6a1, Col9a1, Col10a1, Col14a1, Col15a1, and Col27a1; and the cell cycle regulatory genes Ccnb1, Ccnb2, Skal, and Cdk1. The DE genes associated with the response to wounding and ECM terms exhibited a mixture of up- and downregulation in the scar, compared with naïve, VFF condition; in contrast, 29 of $30 \mathrm{DE}$ genes associated with the cell division term were upregulated in the scar VFF condition. Overall, these transcriptomic data indicate clear system-level differences between scar and naïve VFFs at P1.

\section{Transcriptome-Level Comparison of In Vitro and In Vivo Scar Models}

Next, we compared the P1 naïve and scar VFF transcriptomes with previously reported in vivo data from naïve and scarred VF mucosae. ${ }^{28}$ These in vivo data were obtained using an identical rat strain and age at the time of VF injury, an identical surgical procedure and scar maturation period, and identical sample processing and microarray protocols. Comparisons of probes and genes that were DE in the scar, compared with naïve, conditions in both in vitro and in vivo models showed limited overlap: 18 probes, corresponding to 14 genes, exhibited DE in both models (Figure 5a). These transcriptome-level differences were further emphasized by an analysis of naïve VFF compared with naïve VF mucosa, as well scar VFF compared with scar VF mucosa (Figure 5b). Both analyses revealed a substantial number of $\mathrm{DE}$ probes ( $>10000$ in both naïve and scar comparisons) and genes ( $>6000$ in both naïve and scar comparisons) across experimental systems. Follow-up evaluation of relative expression levels revealed a number of wound healing, fibrosis and ECM-related genes (including the previously evaluated Col1a1 and Acta2 genes) that were highly upregulated $\left(\log _{2}\right.$ fold change $\left.>3\right)$ in the in vitro, compared with in vivo, condition (Figure 5c). Enrichment analysis of the $6700 \mathrm{DE}$ genes in the in vitro vs in vivo scar comparison highlighted an array of biological functions consistent with the system-wide repair program and involvement of epithelial and endothelial cells, myocytes, leukocytes, and neurons in vivo (Supplementary Table S2).

\section{Effect of Growth Factor Stimulation on Scar-Related Transcriptional Activity}

Given our data showing clear phenotypic differences between naïve and scar VFFs at P1, we evaluated the responsiveness of scar VFFs to stimulation with exogenous HGF and TGF- $\beta 3$. These biologics have shown therapeutic potential when delivered to naïve VFFs in vitro, ${ }^{17,29,47,48}$ as well as when delivered to injured or scarred VF mucosae in vivo. ${ }^{17,30,49-51}$ Treatment with HGF downregulated Colla1 and Acta2 transcription in a dose-dependent manner $(P<0.05$; Figure $6 \mathrm{a}$ and $\mathrm{b})$. Treatment with TGF- $\beta 3$ had no effect on Colla1 $(P>0.05$; Figure 6a) but upregulated Acta2 transcription at all doses $(P<0.05$; Figure $6 \mathrm{~b})$. These data show that $\mathrm{P} 1$ scar VFFs are amenable to manipulation using growth factors, as has been reported for their naïve counterparts.

\section{DISCUSSION}

In this study, we pursued detailed characterization of scar VFFs obtained from surgically injured rat VF mucosae, 
compared with VFFs from experimentally naïve, age-matched tissue. The purpose of our experiments was to outline the parameters of an easily replicated in vitro model for ongoing preclinical VF fibrosis research. Compared with naïve cells, scar VFFs exhibited increased proliferation, increased Colla1
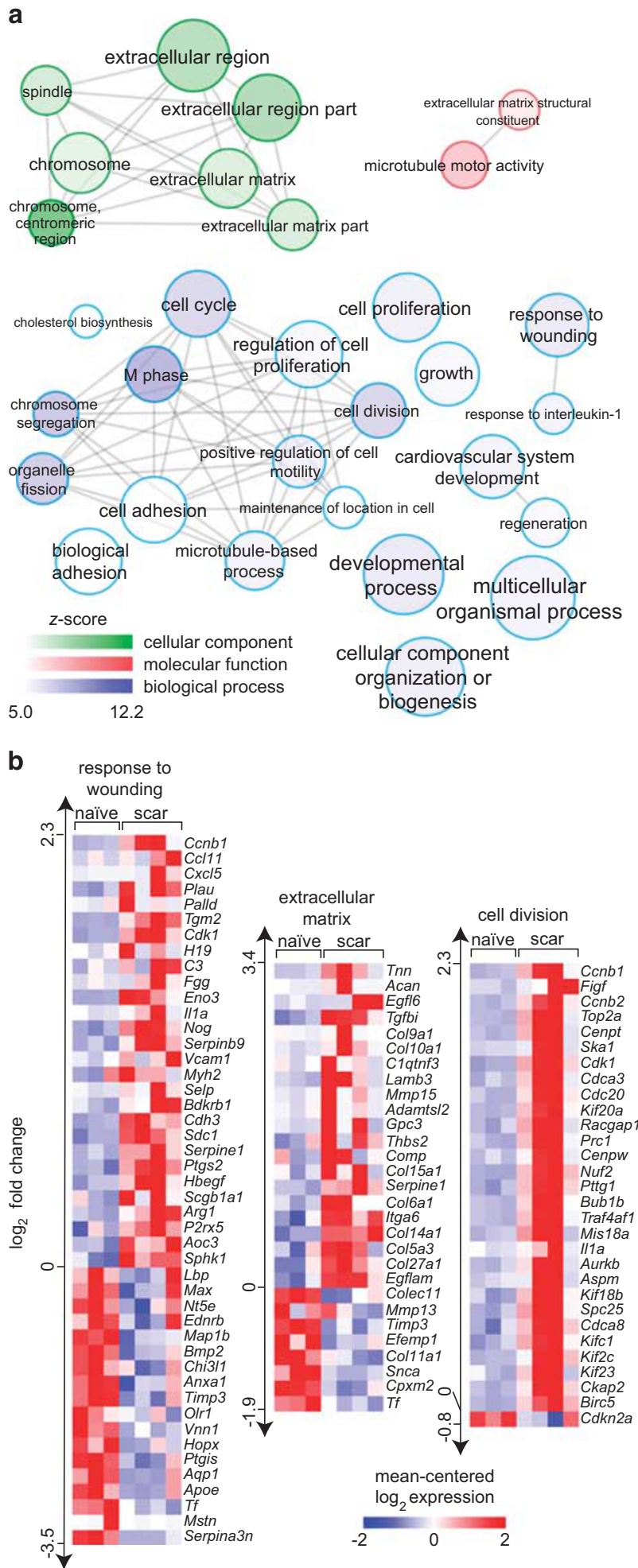

transcription and collagen, type I synthesis, increased Acta2 transcription and $\alpha$-SMA synthesis, and enhanced contractile function. These features, which were predominantly associated with P1 cells, suggest that VFFs isolated from mature scar tissue hold a myofibroblast phenotype, despite histologic, ${ }^{13}$ and transcriptomic ${ }^{28}$ data showing that most wound healing events are completed by this time point in vivo.

Normally, contractile myofibroblasts are removed by apoptosis during the final phase of wound healing, leaving a residual population of quiescent fibroblasts at the site of injury. ${ }^{4}$ In some situations, however, myofibroblasts persist at the wound site and disrupt the resolution of wound healing, resulting in pathologic outcomes such as hypertrophic and keloidal scarring. ${ }^{52,53}$ Given the identification of P1 VFFs with a contractile myofibroblastic phenotype in this study, our model appears to represent such persistently (and pathologically) activated VF mucosal cells. Despite showing no difference in Col3a1 and Fn1 transcription, P1 scar VFFs synthesized excess collagen, type I compared with naïve VFFs, a hallmark of fibrotic disorders and a feature of cultured myofibroblasts isolated from multiple tissue sources. ${ }^{54-56}$ In addition, P1-P3 scar VFFs proliferated more rapidly than P1-P3-naïve VFFs. Previous studies of cultured fibroblasts and myofibroblasts isolated from wounded or pathologically fibrotic skin have reported conflicting proliferation data, however it is important to note that, with the exception of one study, ${ }^{57}$ data were collected from cells at P3 and beyond. ${ }^{58-60}$ In the VF literature, Kumai et al ${ }^{20}$ reported increased proliferation in P3 scar VFFs isolated from electrocauterized ferret VF mucosae compared with controls, whereas Jetté et al ${ }^{22}$ reported reduced proliferation in P5 scar VFFs isolated from a single human patient, compared with control cells from an age- and sex-matched donor. These discrepant findings might reflect methodological differences, such as the type of injury used to induce scarring, the culture passage used for in vitro experiments, or the passage technique itself (eg, differences in split ratio and plating density).

Our transcriptomic analysis of naïve and scar VFFs at P1 corroborated our initial experimental data and provided

Figure 4 Analysis of the scar VFF transcriptome. (a) Gene ontology-based enrichment analysis of DE genes in scar, compared with naïve, VFFs at P1. Enriched ontology terms are depicted as nodes; highly similar terms are connected by edges. Cellular component terms are green; molecular function terms are red; biological process terms are blue. Node color intensity corresponds to the $Z$-score associated with term enrichment. Node and label font size are proportional to the generality of the term in the underlying ontology. (b) Heat maps showing mean-centered $\log _{2^{-}}$ expression data for DE genes associated with the response to wounding, extracellular matrix, and cell division ontology terms. DE genes are ranked by $\log _{2}$ fold change (scar normalized to naïve) along the vertical axis. This experiment was performed with $n=3$ biological replicates in the naïve condition and $n=4$ biological replicates in the scar condition. 
a
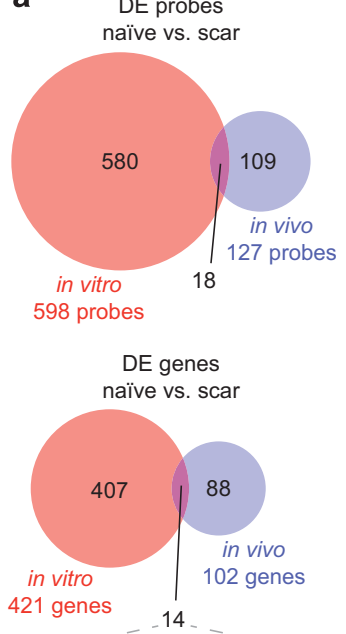

Acan Comp Rassf4

Cc/9 Myh2 Serpina3n

Cldn11 Npas2 Serpine1

Col10a1 Per2 Slc2a6

Col9a1 Ptgis

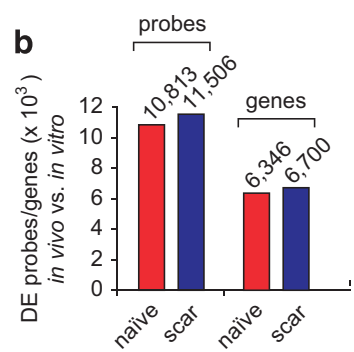

Figure 5 Transcriptome-level comparison of in vitro and in vivo scar models. (a) Venn diagrams showing limited overlap in the DE probe set/gene set identified in scar VFFs compared with naïve cells (at P1), and the DE probe set/gene set identified in scarred VF mucosa compared with naïve mucosa. Fourteen genes were identified as DE in both experimental systems. (b) Summary of DE probes/genes identified in direct in vivo vs in vitro comparisons of naïve VFFs and naïve VF mucosa, and scar VFF and scarred VF mucosa. (c) Heat maps showing meancentered $\log _{2}$-expression data for the $6346 \mathrm{DE}$ genes identified in naïve VFF vs naïve VF mucosa, and the 6700 DE genes identified in scar VFF vs scarred VF mucosa. DE genes are ranked by $\log _{2}$ fold change (in vitro normalized to in vivo) along the vertical axis. The annotations indicate a subset of DE genes associated with wound healing, fibrosis and ECM that were highly upregulated in the in vitro, compared with in vivo, condition ( $\log _{2}$ fold change $>3$ ) for both naïve and scar comparisons. All experiments were performed with $n=3-5$ biological replicates per condition.

additional characterization of the major phenotypic differences between these cell types. Scar VFFs exhibited a series of DE genes associated with the wound response, as well as ECM regulation and cell proliferation. Many of these DE genes represent pathways and targets with potential experimental and therapeutic relevance for future studies. In contrast, with the relatively clear biological function inferred from these in vitro data, our follow-up comparison of naïve and scar VFFs with naïve and scarred VF mucosae revealed marked
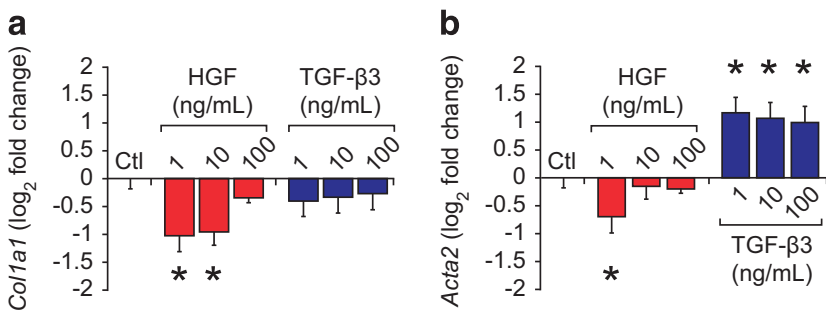

Figure 6 Effect of growth factor stimulation on scar-related transcriptional activity. Exogenous HGF stimulation downregulated Col1a1 (a) and Acta2 (b) transcription in scar VFFs at P1 in a dose-dependent manner, whereas exogenous TGF- $\beta 3$ stimulation upregulated Acta2 transcription only (b). Data are presented as mean fold change \pm s.e.m. vs the untreated control $(\mathrm{Ctl})$ condition and are $\log _{2}$-transformed to best represent bidirectional stimulation effects. All experiments were performed with $n=4$ biological replicates per condition. ${ }^{*} P<0.05$.

transcriptomic differences ( $>6000 \mathrm{DE}$ genes in both naïve and scar comparisons) across model systems. These differences are not surprising, however, as VF mucosa: (i) contains substantially more VF epithelial cells than VFFs, ${ }^{25,61}$ and (ii) is subject to systemic influences in vivo, ${ }^{62}$ which differs from the isolated environment of VFFs in vitro. The in vitro system does not represent the full biological complexity of the VF mucosa, but rather provides an experimental tool to investigate discrete mechanisms that impact its function. Our findings reiterate that these in vitro and in vitro models offer complementary, but not interchangeable, approaches to studying VF fibrosis.

As noted above, we observed clear differences between naïve and scar VFFs at P1 followed by a coalescence of the scar and naïve VFF phenotypes at later passages. This pattern was particularly evident in the proliferation, ECM production and myofibroblast marker assays and led us to use P1 cells exclusively for the gel contraction and transcriptomic assays, as well as the growth factor stimulation experiments. Variation in ECM-related transcriptional activity across culture passages has been previously reported in naïve VFFs. ${ }^{31}$ Our finding in scar VFFs suggests that these cells, which exhibit a convincing myofibroblast phenotype at P1, undergo culture-associated dedifferentiation when outside of their in vivo tissue niche and eventually reassume a quiescent phenotype. Therefore, P1 cells may be most appropriate for conducting fibrosis-related experiments. This conclusion raises practical considerations, however, as conducting experiments at $\mathrm{P} 1$ gives limited opportunity for expanding cell numbers and, by extension, limited opportunity to use an individual animal's cells for multiple experiments and multiple assays per experiment.

As scar VFFs appear to hold a transient phenotype under standard culture conditions, it would be helpful to identify the molecular parameters needed to attenuate or prevent their dedifferentiation to a quiescent state. It is well established that TGF- $\beta 1$ stimulation induces myofibroblast differentiation in naïve fibroblasts (including in naïve VFFs); ${ }^{17,63,64}$ however, it 
is currently unknown if these culture-induced myofibroblasts are equivalent to those isolated from fibrotic tissue. Beyond inducing and maintaining a scar VFF phenotype, directing these cells from a scar to a naive, quiescent phenotype has additional experimental (and therapeutic) value. Previous work has shown myofibroblast dedifferentiation following treatment with amniotic membrane stromal extract, ${ }^{65}$ as well as with fibroblast growth factor plus heparin. ${ }^{66}$ Here, we observed reduced Col1a1 and Acta2 transcription by P1 scar VFFs following low-dose HGF treatment, consistent with data from naïve VFFs, ${ }^{29,47,48}$ late passage scar VFFs, ${ }^{21}$ and injured or scarred VF mucosae. ${ }^{49-51}$ We also observed increased Acta2 transcription following TGF- $\beta 3$ treatment, which aligns with data from naïve VFFs showing that although TGF- $\beta 3$ is capable of promoting myofibroblast differentiation, it is substantially less potent than the profibrotic isoforms TGF- $\beta 1$ and TGF- $\beta 2 .{ }^{17}$

In summary, our data characterize key phenotypic features of rat scar VFFs at the transcript, transcriptome, protein, and functional levels. Despite the practical limitations inherent to working with early passage cells, this experimental model is easily implemented in any suitably equipped laboratory and has the potential to improve the applicability of preclinical VF fibrosis research.

Supplementary Information accompanies the paper on the Laboratory Investigation website (http://www.laboratoryinvestigation.org)

\section{ACKNOWLEDGMENTS}

We gratefully acknowledge Glen Leverson (Department of Surgery, University of Wisconsin School of Medicine and Public Heath, Madison, WI) for consultation on the parametric statistical testing. This work was funded by grants from the National Institute on Deafness and other Communication Disorders (grant numbers R01 DC004428, R01 DC010777); and the Clinical and Translational Science Award (CTSA) program of the National Center for Research Resources (grant number UL1 RR025011).

\section{DISCLOSURE/CONFLICT OF INTEREST}

The authors declare no conflict of interest.

1. Flavell SJ, Hou TZ, Lax $S$ et al. Fibroblasts as novel therapeutic targets in chronic inflammation. Br J Pharmacol 2008;153(Suppl 1):S241-S246.

2. Iwaisako K, Brenner DA, Kisseleva T. What's new in liver fibrosis? The origin of myofibroblasts in liver fibrosis. J Gastroenterol Hepatol 2012;27(Suppl 2):65-68.

3. Quaggin $\mathrm{SE}$, Kapus A. Scar wars: mapping the fate of epithelialmesenchymal-myofibroblast transition. Kidney Int 2011;80:41-50.

4. Hinz B, Phan SH, Thannickal VJ et al. Recent developments in myofibroblast biology: paradigms for connective tissue remodeling. Am J Pathol 2012;180:1340-1355.

5. Hinz B, Phan SH, Thannickal VJ et al. The myofibroblast: one function, multiple origins. Am J Pathol 2007;170:1807-1816.

6. Desmoulière A, Darby IA, Gabbiani G. Normal and pathologic soft tissue remodeling: role of the myofibroblast, with special emphasis on liver and kidney fibrosis. Lab Invest 2003;83:1689-1707.

7. Bataller R, Brenner DA. Liver fibrosis. J Clin Invest 2005;115:209-218.

8. Shimizu K, Yoshizato K. Organ-dependent expression of differentiated states in fibroblasts cultured in vitro. Dev Growth Differ 1992;34:43-50.

9. Hirano $M$, Sato $K$, Nakashima T. Fibroblasts in human vocal fold mucosa. Acta Otolaryngol 1999;119:271-276.
10. Chan RW, Titze IR. Viscoelastic shear properties of human vocal fold mucosa: measurement methodology and empirical results. J Acoust Soc Am 1999;106:2008-2021.

11. Gray SD, Titze IR, Chan RW et al. Vocal fold proteoglycans and their influence on biomechanics. Laryngoscope 1999;109:845-854.

12. Rousseau B, Hirano S, Scheidt TD et al. Characterization of vocal fold scarring in a canine model. Laryngoscope 2003;113:620-627.

13. Tateya T, Tateya I, Sohn JH et al. Histologic characterization of rat vocal fold scarring. Ann Otol Rhinol Laryngol 2005;114:183-191.

14. Hirano S, Minamiguchi S, Yamashita M et al. Histologic characterization of human scarred vocal folds. J Voice 2009;23:399-407.

15. Welham NV, Choi SH, Dailey SH et al. Prospective multi-arm evaluation of surgical treatments for vocal fold scar and pathologic sulcus vocalis. Laryngoscope 2011;121:1252-1260.

16. Suehiro A, Hirano S, Kishimoto $\mathrm{Y}$ et al. Effects of basic fibroblast growth factor on rat vocal fold fibroblasts. Ann Otol Rhinol Laryngol 2010;119: 690-696.

17. Chang $Z$, Kishimoto $Y$, Hasan $A$ et al. TGF- $\beta 3$ modulates the inflammatory environment and reduces scar formation following vocal fold mucosal injury in rats. Dis Model Mech 2014;7:83-91.

18. Chen X, Thibeault SL. Biocompatibility of a synthetic extracellular matrix on immortalized vocal fold fibroblasts in 3-D culture. Acta Biomater 2010;6:2940-2948.

19. King $\mathrm{SN}$, Hanson $\mathrm{SE}$, Chen $X$ et al. In vitro characterization of macrophage interaction with mesenchymal stromal cell-hyaluronan hydrogel constructs. J Biomed Mater Res A 2014;102:890-902.

20. Kumai $\mathrm{Y}$, Kobler JB, Park $\mathrm{H}$ et al. Crosstalk between adipose-derived stem/stromal cells and vocal fold fibroblasts in vitro. Laryngoscope 2009;119:799-805.

21. Kumai $Y$, Kobler JB, Park $\mathrm{H}$ et al. Modulation of vocal fold scar fibroblasts by adipose-derived stem/stromal cells. Laryngoscope 2010;120:330-337.

22. Jetté ME, Hayer SD, Thibeault SL. Characterization of human vocal fold fibroblasts derived from chronic scar. Laryngoscope 2013;123:738-745.

23. King SN, Berchtold CM, Thibeault SL. Lipopolysaccharide responsiveness in vocal fold fibroblasts. J Inflamm 2014;11:42.

24. King SN, Chen F, Jetté ME et al. Vocal fold fibroblasts immunoregulate activated macrophage phenotype. Cytokine 2013;61:228-236.

25. Ling C, Yamashita $M$, Waselchuk EA et al. Alteration in cellular morphology, density and distribution in rat vocal fold mucosa following injury. Wound Repair Regen 2010;18:89-97.

26. Tateya I, Tateya T, Lim X et al. Cell production in injured vocal folds: a rat study. Ann Otol Rhinol Laryngol 2006;115:135-143.

27. Welham NV, Montequin DW, Tateya I et al. A rat excised larynx model of vocal fold scar. J Speech Lang Hear Res 2009;52:1008-1020.

28. Welham NV, Ling C, Dawson JA et al. Microarray-based characterization of differential gene expression during vocal fold wound healing in rats. Dis Model Mech 2015;8:311-321.

29. Kishimoto $Y$, Hirano $S$, Suehiro A et al. Effect of exogenous hepatocyte growth factor on vocal fold fibroblasts. Ann Otol Rhinol Laryngol 2009;118:606-611.

30. Ohno S, Hirano S, Kanemaru S-I et al. Transforming growth factor $\beta 3$ for the prevention of vocal fold scarring. Laryngoscope 2012;122: 583-589.

31. Thibeault SL, Li W, Gray SD et al. Instability of extracellular matrix gene expression in primary cell culture of fibroblasts from human vocal fold lamina propria and tracheal scar. Ann Otol Rhinol Laryngol 2002;111: 8-14.

32. Thibeault SL, Li W, Bartley S. A method for identification of vocal fold lamina propria fibroblasts in culture. Otolaryngol Head Neck Surg 2008:139:816-822.

33. Lincoln DW, Whitney RG, Smith JR. In vitro proliferation and lifespan of bovine aorta endothelial cells: response to conditioned media. J Cell Sci 1982;56:281-292.

34. Abe R, Donnelly SC, Peng $T$ et al. Peripheral blood fibrocytes: differentiation pathway and migration to wound sites. J Immunol 2001;166:7556-7562.

35. Chang $Z$, Ling $C$, Yamashita $M$ et al. Microarray-driven validation of reference genes for quantitative real-time polymerase chain reaction in a rat vocal fold model of mucosal injury. Anal Biochem 2010;406: 214-221.

36. Schneider CA, Rasband WS, Eliceiri KW. NIH Image to ImageJ: 25 years of image analysis. Nat Methods 2012;9:671-675. 
37. R Development Core Team. R: a Language and Environment for Statistical Computing. R Foundation for Statistical Computing: Vienna, 2007.

38. Irizarry RA, Hobbs B, Collin F et al. Exploration, normalization, and summaries of high density oligonucleotide array probe level data. Biostat 2003;4:249-264.

39. Wu Z, Irizarry RA. Preprocessing of oligonucleotide array data. Nat Biotechnol 2004:22:656-658.

40. Kendziorski CM, Newton MA, Lan $\mathrm{H}$ et al. On parametric empirical Bayes methods for comparing multiple groups using replicated gene expression profiles. Stat Med 2003;22:3899-3914.

41. Newton MA, Quintana FA, Boon den JA et al. Random-set methods identify distinct aspects of the enrichment signal in gene-set analysis. Ann Appl Stat 2007;1:85-106.

42. Gene Ontology Consortium. Gene Ontology annotations and resources. Nucleic Acids Res 2013;41:D530-D535.

43. Supek $F$, Bošnjak $M$, Škunca $N$ et al. REVIGO summarizes and visualizes long lists of gene ontology terms. PLoS One 2011;6:e21800.

44. Cline MS, Smoot M, Cerami E et al. Integration of biological networks and gene expression data using Cytoscape. Nat Protoc 2007;2: 2366-2382.

45. Pavlidis $P$, Noble WS. Matrix2png: a utility for visualizing matrix data. Bioinformatics 2003;19:295-296.

46. Iwano $\mathrm{M}$, Fischer $\mathrm{A}$, Okada $\mathrm{H}$ et al. Conditional abatement of tissue fibrosis using nucleoside analogs to selectively corrupt DNA replication in transgenic fibroblasts. Mol Ther 2001;3:149-159.

47. Hirano S, Bless DM, Heisey DM et al. Effect of growth factors on hyaluronan production by canine vocal fold fibroblasts. Ann Otol Rhinol Laryngol 2003;112:617-624.

48. Hirano S, Bless DM, Heisey DM et al. Roles of hepatocyte growth factor and transforming growth factor beta1 in production of extracellular matrix by canine vocal fold fibroblasts. Laryngoscope 2003;113:144-148.

49. Hirano S, Bless DM, Nagai $\mathrm{H}$ et al. Growth factor therapy for vocal fold scarring in a canine model. Ann Otol Rhinol Laryngol 2004;113:777-785.

50. Hirano S, Bless DM, Rousseau B et al. Prevention of vocal fold scarring by topical injection of hepatocyte growth factor in a rabbit model. Laryngoscope 2004;114:548-556.

51. Kishimoto $Y$, Hirano S, Kitani $Y$ et al. Chronic vocal fold scar restoration with hepatocyte growth factor hydrogel. Laryngoscope 2010;120: 108-113.

52. Ding J, Hori K, Zhang $\mathrm{R}$ et al. Stromal cell-derived factor 1 (SDF-1) and its receptor CXCR4 in the formation of postburn hypertrophic scar (HTS). Wound Repair Regen 2011;19:568-578.
53. Ding J, Ma Z, Liu $\mathrm{H}$ et al. The therapeutic potential of a C-X-C chemokine receptor type 4 (CXCR-4) antagonist on hypertrophic scarring in vivo. Wound Repair Regen 2014;22:622-630.

54. Fritz D, Cai L, Stefanovic L et al. Progress towards discovery of antifibrotic drugs targeting synthesis of type I collagen. Curr Med Chem 2011;18:3410-3416.

55. Poobalarahi F, Baicu CF, Bradshaw AD. Cardiac myofibroblasts differentiated in 3D culture exhibit distinct changes in collagen production, processing, and matrix deposition. Am J Physiol Heart Circ Physiol 2006;291:H2924-H2932.

56. Phan SH. Biology of fibroblasts and myofibroblasts. Proc Am Thorac Soc 2008;5:334-337.

57. Vande Berg JS, Rudolph R, Poolman WL et al. Comparative growth dynamics and actin concentration between cultured human myofibroblasts from granulating wounds and dermal fibroblasts from normal skin. Lab Invest 1989;61:532-538.

58. Vande Berg JS, Rudolph R, Woodward M. Comparative growth dynamics and morphology between cultured myofibroblasts from granulating wounds and dermal fibroblasts. Am J Pathol 1984;114: 187-200.

59. Ladin DA, Hou Z, Patel D et al. p53 and apoptosis alterations in keloids and keloid fibroblasts. Wound Repair Regen 1998;6:28-37.

60. Lanni C, Bottone MG, Bardoni A et al. Proliferation characteristics and polyploidization of cultured myofibroblasts from a patient with fibroblastic rheumatism. Eur J Histochem 2003;47:257-262.

61. Ling C, Raasch JL, Welham NV. E-cadherin and transglutaminase-1 epithelial barrier restoration precedes type IV collagen basement membrane reconstruction following vocal fold mucosal injury. Cells Tissues Organs 2011;193:158-169.

62. Ling C, Yamashita M, Zhang J et al. Reactive response of fibrocytes to vocal fold mucosal injury in rat. Wound Repair Regen 2010;18:514-523.

63. Hinz B, Celetta G, Tomasek JJ et al. Alpha-smooth muscle actin expression upregulates fibroblast contractile activity. Mol Biol Cell 2001;12:2730-2741.

64. Vyas B, Ishikawa K, Duflo SM et al. Inhibitory effects of hepatocyte growth factor and interleukin- 6 on transforming growth factor- $\beta 1$ mediated vocal fold fibroblast-myofibroblast differentiation. Ann Otol Rhinol Laryngol 2010;119:350-357.

65. Li W, He H, Chen Y-T et al. Reversal of myofibroblasts by amniotic membrane stromal extract. J Cell Physiol 2008;215:657-664.

66. Maltseva O, Folger $\mathrm{P}$, Zekaria D et al. Fibroblast growth factor reversal of the corneal myofibroblast phenotype. Invest Ophthalmol Vis Sci 2001;42:2490-2495. 